\title{
LETTER TO THE EDITOR Circular mating as an option for the genetic management of captive populations: response to Caballero et al.
}

\author{
Heredity (2017) 119, 49-50; doi:10.1038/hdy.2017.16; published online 22 March 2017
}

In their work, Caballero et al. (2016) assess the efficiency of circular sib mating $(\mathrm{CM})$ for the genetic management of captive populations. They argue that if one adopts findings on wild populations suggesting that the number of lethal equivalents per genome $(B)$ are at least six, CM performs poorly, and 'should be avoided because it would lead to unacceptably high extinction risk'. We agree that CM would increase extinction risk when populations harbour high inbreeding depression and/or have a low reproductive output. However, we advance three major arguments suggesting that the relevant number of lethal equivalents for population persistence should be, in many cases, significantly lower emphasizing the relevance of $\mathrm{CM}$ as a breeding strategy in ex situ genetic management.

1. Inbreeding depression is typically less severe under benign captive conditions than under stressful wild ones.

Crnokrak and Roff (1999) reported that inbreeding depression was seven times higher in the wild than in captivity. In salmonids, egg to smolt survival is typically $85-95 \%$ in hatcheries against $1-5 \%$ in the wild (Reisenbichler et al., 2004). Moreover, the meta-analysis of Fox and Reed (2011) revealed a strong inbreeding-environment interaction with inbreeding depression being significantly lower in relatively benign than in stressful conditions.

Recessive alleles that would be lethal in the wild when exposed in the homozygote form, such as albinism or some forms of epilepsy, may be far less detrimental to captive individuals. In these cases, the effects of close inbreeding should disappear if reintroduction is preceded by a single generation of random mating.

The authors take partially into account the relaxation of selection in captivity by assuming that selection is halved for only non-lethal mutations relatively to the wild. However, according to the evidence listed above, inbreeding depression in their simulations could be exaggerated in some cases.

2. Purging of inbreeding depression occurs in small populations. Although inbreeding depression can be highly variable among populations (Boakes et al., 2007), populations with a history of size decline or bottlenecking could conceal smaller inbreeding depression than the ancestral populations, due to previous purging (Pujol et al., 2009; Facon et al., 2011; Laws and Jamieson, 2011). For this reason, Garcia-Dorado (2015) suggested that 'when predicting the short-term impact of inbreeding on fitness, it should be taken into account that many endangered populations may have inbreeding depression substantially smaller than $B=6$ '. Yet, the arguments of the authors against
$\mathrm{CM}$ are almost solely based on the assumption of such a high number of lethal equivalents.

3. A part of inbreeding depression is due to the expression of traits that are marginally relevant to population viability, such as (i) later life-history traits (Charlesworth and Hughes, 1996; Moorad and Hall, 2009), and (ii) traits associated to sexual selection.

Mating preferences, for instance, can establish a trait even if it has negative side effects on individual survival and hence on population viability, such as extravagant male traits (Dieckmann and Ferrière, 2004).

Although such traits are reported to show high inbreeding depression, this part of inbreeding depression has a weak influence on population dynamics and should not be included in population viability considerations. Hence, the relevant number of lethal equivalents for persistence and, therefore, the negative effects associated with $\mathrm{CM}$ could be in some cases considerably lower than assumed by the authors.

\section{PERSPECTIVES}

These arguments suggest that inbreeding depression relevant for the survival of captive populations may be lower than assumed by the authors. CM may, therefore, be applicable for many captive populations that are under weak selection or have a prior history of purging. In such cases, $\mathrm{CM}$ reaches the goals of genetic management more efficiently than methods of inbreeding avoidance or equalization of parental contributions (Theodorou and Couvet, 2010; Theodorou and Couvet, 2015). More precisely, CM: (a) retains higher allelic diversity while it increases adaptive variation during the sojourn in captivity, (b) halves the rate of adaptation to captive conditions, and (c) hinders the accumulation of deleterious alleles.

Finally, an additional argument concerns the consistency of the mutation model chosen. Mutation-accumulation experiments yield contrasting results considering either (i) few mutations $(\lambda=0.01-$ $0.03)$ of large average detrimental effect, $\bar{s}=0.1-0.2$ and low dominance, $\bar{h}=0.1-0.2$, or (ii) frequent mutations with small average effect and higher dominance, $\lambda=0.5-1, \bar{s}=0.02-0.05$, $\bar{h}=0.3-0.4$ (Perez-Figueroa et al., 2009). The authors mix the two contrasting models by using the dominance coefficient of the former along with the mutation rate and detrimental effect of the latter. This is problematic in two aspects: First, if the former model is fully adopted, the effect of deleterious mutations on population viability should be negligible (Fernández and Caballero, 2001). Second, if the dominance coefficient follows the latter model, a higher mutation rate must be assumed to produce the desired number of lethal equivalents, $B=6$. Such large mutation rates are 
suggested for long-lived organisms but rarely reported. Hence, the model of Caballero et al. (2016) is to some extent speculative and contradictory with some of the empirical facts.

Hence, CM may be adopted during the first generations in captivity, especially to preserve adaptive variation. Reversing to other strategies is possible and could be advantageous; a single generation of panmixia, for instance, can alleviate the negative fitness effects of close inbreeding (Theodorou and Couvet, 2015) with obvious implications for reintroductions. However, further investigation on the effects of combining breeding systems is needed in the light of the points emphasized by Caballero et al. (2016).

Based on the evidence for variation in the extent of inbreeding depression and its effects on the viability of captive populations, we shouldn't rule out CM as an effective management option. Even if inbreeding depression may not pose a serious threat for some captive populations, loss of genetic diversity and adaptation to captivity will still do. Concerning the latter two goals, theory predicts that CM outperforms management options based on inbreeding avoidance. Experimental validation would help assessing the suitability of CM for captive breeding.

\section{CONFLICT OF INTEREST}

The authors declare no conflict of interest.

K Theodorou ${ }^{1}$ and D Couvet ${ }^{2}$ ${ }^{1}$ Biodiversity Conservation Laboratory, Department of Environment, University of the Aegean, Mytilene, Greece and ${ }^{2} U M R 5173$ MNHN-CNRS-UPMC, CP 51, Paris, France E-mail: ktheo@aegean.gr
Boakes EH, Wang J, Amos W (2007). An investigation of inbreeding depression and purging in captive pedigreed populations. Heredity 98: 172-182.

Caballero A, Bravo I, Wang J (2016). Inbreeding load and purging: implications for the short-term survival and the conservation management of small populations. Heredity 118: $177-185$.

Charlesworth B, Hughes KA (1996). Age-specific inbreeding depression and components of genetic variance in relation to the evolution of senescence. Proc Natl Acad Sci USA 93: 6140-6145.

Crnokrak P, Roff DA (1999). Inbreeding depression in the wild. Heredity 83: 260-270.

Dieckmann U, Ferrière R (2004). Adaptive dynamics and evolving biodiversity. In: Ferrière R, Dieckmann U, Couvet D (eds). Evolutionary Conservation Biology. Cambridge University Press: Cambridge, UK, pp 188-224.

Facon B, Hufbauer RA, Tayeh A, Loiseau A, Lombaert E, Vitalis R et al. (2011). Inbreeding depression is purged in the invasive insect Harmonia axyridis. Curr Biol 21: 424-427.

Fernández J, Caballero A (2001). A comparison of management strategies for conservation with regard to population fitness. Conserv Genet 2: 121-131.

Fox CW, Reed DH (2011). Inbreeding depression increases with environmental stress: an experimental study and meta-analysis. Evolution 65: 246-258.

Garcia-Dorado A (2015). On the consequences of ignoring purging on genetic recommendations for minimum viable population rules. Heredity 115: 185-187.

Laws RJ, Jamieson IG (2011). Is lack of evidence of inbreeding depression in a threatened New Zealand robin indicative of reduced genetic load? Anim Conserv 14: 47-55.

Moorad JA, Hall DW (2009). Age-dependent mutational effects curtail the evolution of senescence by antagonistic pleiotropy. J Evol Biol 22: 2409-2419.

Perez-Figueroa A, Caballero A, Garcia-Dorado A, Lopez-Fanjul C (2009). The action of purifying selection, mutation and drift on fitness epistatic systems. Genetics 183: 299-313.

Pujol B, Zhou SR, Vilas JS, Pannell JR (2009). Reduced inbreeding depression after species range expansion. Proc Natl Acad Sci USA 106: 15379-15383.

Reisenbichler R, Rubin SP, Wetzel S, Phelps S (2004). Natural selection after release from a hatchery leads to domestication in steelhead, Oncorhynchus mykiss. In: Leber KM, Blackenship HL, Kitada S, Svasand T (eds). Stock Enhancement and Sea zRanching: Developments Pitfalls and Opportunities. Blackwell Science: Oxford, UK, pp 371-383.

Theodorou K, Couvet D (2010). Genetic management of captive populations: the advantages of circular mating. Conserv Genet 11: 2289-2297.

Theodorou K, Couvet D (2015). The efficiency of close inbreeding to reduce genetic adaptation to captivity. Heredity 114: 38-47. 